\title{
Microwave Heating Induced On-Demand Droplet Generation in Microfluidic Systems
}

\author{
Weijia Cui, Gurkan Yesiloz, and Carolyn L. Ren*
}

Cite This: Anal. Chem. 2021, 93, 1266-1270

Read Online

\section{ACCESS}

山ll Metrics \& More

ABSTRACT: In this note, we report a simple, new method for droplet generation in microfluidic systems using integrated microwave heating. This method enables droplet generation ondemand by using microwave heating to induce Laplace pressure change at the interface of the two fluids. The distance between the interface and junction and microwave excitation power have been found to influence droplet generation. Although this method is limited in generating droplets with a high rate, the fact that it can be integrated with microwave sensing that can be used as the feedback to tune the supply flow of materials presents unique advantages for applications that require dynamic tuning of material properties in droplets.

\section{INTRODUCTION}

Droplet microfluidics has drawn ever-increasing attention from both academia and industry with numerous studies reported on both fundamental research and practical applications ${ }^{1,2}$ over the past two decades with some companies such as Dolomite Bio catching up the developed innovations. ${ }^{3}$ Because droplets are isolated from each other and from the channel walls by the carrier fluid, each individual droplet can serve as an encapsulated cargo platform for cells, particles, or biomolecules (DNA, proteins) and can be a microreactor for chemical or biochemical synthesis. ${ }^{4,5}$ Major advantages of using isolated pico- to nanoliter droplets as reaction vesicles instead of traditional well plates or single-phase microfluidic devices include reduced reagent consumption, enhanced mixing, unparalleled high throughput, and shortened reaction time. ${ }^{6}$

For most applications, droplet generation marks the initial and important step, which is expected to have stable and predictable outcomes. ${ }^{7}$ A well-controlled droplet generation process is required for various applications, including biochemical screening, click chemistry, and DNA polymerase chain reaction to have precise products and results. ${ }^{8-10}$

Although droplets can be generated passively at $\mathrm{kHz}$ rates in microchannel networks by varying applied pressures and channel geometries, ${ }^{7}$ precise control of individual droplets becomes challenging because any design flaws, fabrication defects, and pressure fluctuations would affect the designed functionality. It is also difficult to generate droplets on-demand which is important for many applications requiring a higher degree control of droplet number and spacing as well as reaction time. ${ }^{11}$

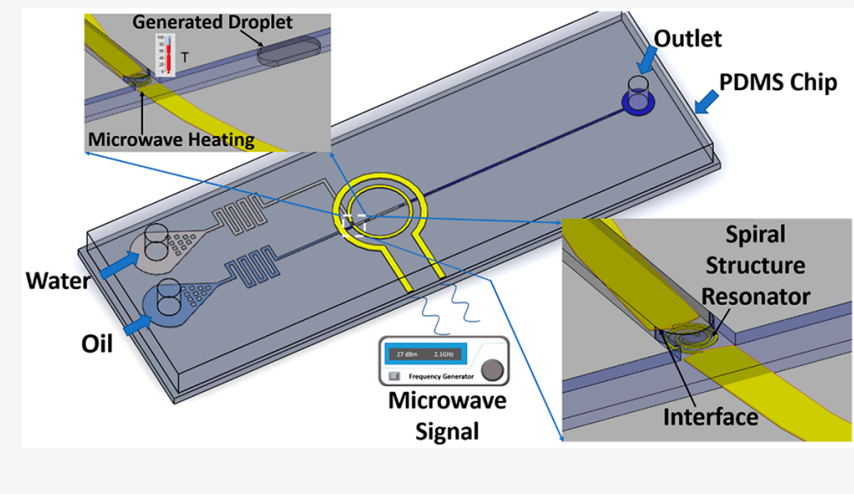

Alternatively, active methods are favorable for on-demand generation of droplets by integrating external forces. For comprehensive understanding of active methods for droplet generation, readers are referred to some excellent reviews. ${ }^{7,12}$ Briefly, some active methods involve the integration of moving parts such as mechanical valves ${ }^{13,14}$ and electrically actuated membranes, ${ }^{15}$ while others rely on the applied electrical fields to control droplet volume and generation frequencies. ${ }^{16-18}$ These electrical methods normally generate an electric field to manipulate droplet generation in microfluidics, which are realized by embedding electrodes to microfluidic devices and then inducing charges accumulating on the water-oil interface. $^{7}$

Droplet generation is mainly dominated by the competition between interfacial tension and viscous forces when flow rates are not high (shear force is negligible), and thus, varying the interfacial tension becomes one of the effective ways for generating droplet on-demand in addition to varying flow and geometric conditions. Interfacial tension is influenced by temperature and surfactants that can be added to one or both phases of the fluids. ${ }^{19}$ Embedded heaters were straightforward for thermally mediated generation of droplets or bubbles. ${ }^{20,21}$ These resistive heating methods have difficulty achieving

Received: October 20, 2020

Accepted: December 16, 2020

Published: December 29, 2020 
localized heating of droplets and have a relatively long response time for switching on/off. Stan et al. reported a method to implement two different temperature zones on a microfluidic flow-focusing generator by using two heat exchangers that are insulated from each other. ${ }^{22}$ They achieved independent control of drop size and velocity by varying both temperature and flow rate. The study did not aim at forming droplets through heating; rather, temperature zones are utilized for manipulation of droplet size.

Cordero et al. later reported the use of a laser beam to generate different optical patterns that were able to localize heating at the desired locations for generating and directing droplets. ${ }^{23}$ Park et al. used laser-induced cavitation to improve the throughput of on-demand droplet generation. ${ }^{24}$ These optical methods offer more control over the droplet generation than the traditional thermal methods with the cost of synchronizing the optical system with the fluid system.

Microwave heating has also been demonstrated for enhancing droplet mixing ${ }^{25}$ and assisting in nanoparticle synthesis. ${ }^{26}$ Microwave heating is basically converting electromagnetic energy to thermal energy deposited in droplets of particular dielectric properties efficiently. ${ }^{27}$ It can selectively heat droplets, leaving the surrounding carrier fluid unheated based on different dielectric properties. ${ }^{28}$ For example, PDMS (chip material) and fluorinated oil (droplet carrier) that are used in this study have a dielectric constant around 2.4 and 1.9, respectively, much lower than that of the water droplets around 78.4 at room temperature. Although PDMS and fluorinated oil are microwave energy absorbers, when the power is supplied at the resonance frequency of the droplet material, the heat absorbed by the PDMS and fluorinated oil is negligible. Moreover, microwave heating exhibits strong localization and features fast heating and cooling, ${ }^{29,30}$ which enables pulse heating, because heating stops once the power is off. This controlled pulse heating provides an opportunity to utilize microwave method as an external source to achieve ondemand droplet generation which is demonstrated in this note. The influence of microwave power on the droplet generation performance is investigated, and the generation mechanism is briefly discussed.

\section{EXPERIMENTAL SECTION}

The reported microwave heater is a cocentric ring resonator made of metal traces on a glass slide that is integrated with a polydimethylsiloxane (PDMS) microfluidic chip, as shown in Figure 1. The outer ring receives power from a frequency

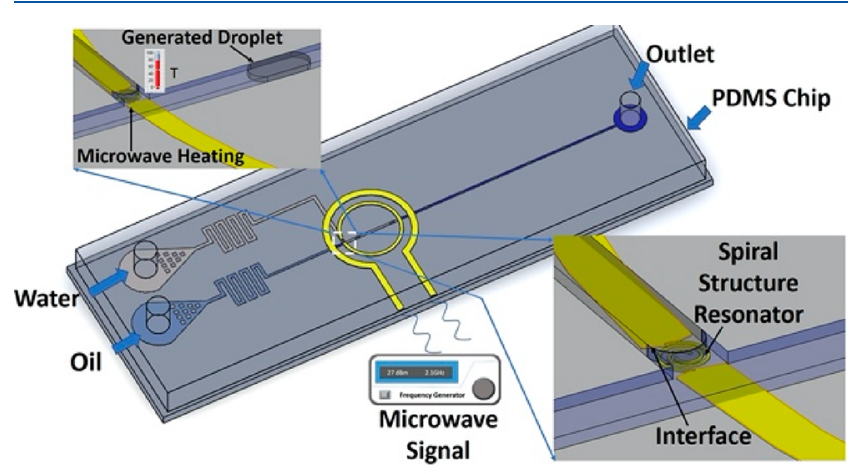

Figure 1. Schematic of the microfluidic chip integrated with the microwave heater. generator (HMC-T2100, Hittite), and the resonating inner ring has a capacitive gap that is aligned with the microfluidic $\mathrm{T}$ junction channel and deposits energy into the liquid at the junction. The fluids including both the carrier phase (fluorinated oil, FC-40 from Sigma-Aldrich) and droplet phase (DI-water) are pumped through the microchannels via a pressure source (Fluigent MFCS- $8 \mathrm{C}$ ), and the droplet generation process is visualized via a CCD camera in a microscope (Eclipse Ti, Nikon, Retiga 2000R, QImaging).

Device Design and Fabrication. The focus of this study is to demonstrate the feasibility of using microwave heating to generate droplets on-demand; therefore, a T-junction generator was chosen because of its simplicity and the superior control it offers over droplet size. The channel width and height are similar to our previous studies on microwave sensing and heating, ${ }^{30}$ which are 210 and $40 \mu \mathrm{m}$, respectively.

The microfluidic chip consists of two parts: a glass slide with the microwave components and a PDMS mold. The detailed fabrication procedures can be found in our previous publication. ${ }^{25}$ Briefly, the microwave components are fabricated using a combination of photolithography and electroplating, while the microchannels are made by using soft-lithography techniques. To ensure the same surface properties for stable droplet formation, a thin PDMS layer is coated on top of the microwave components, serving as one of the channel walls when bonded with the PDMS mold via plasma surface treatment.

\section{RESULTS AND DISCUSSION}

Droplet Generation Mechanism. Although systematic investigation of the mechanism of microwave heating induced on-demand droplet generation is beyond the focus of this study, qualitative discussion is helpful in exploring and designing the system. Most active methods generate droplets by bringing extra energy to disturb the interface. For example, laser mediated droplet generation methods disturb the interface by using the optical cavitation bubble generated with an intense laser pulse focusing on the liquid medium. ${ }^{24}$ Droplets can be generated on-demand by controlling the laser beam, e.g. by controlling either the laser beam power or focusing position. On the contrast, the microwave heater presented here localizes heating to the interface region of the two fluids that aligns with the capacitive gap of the resonator. The temperature increase in the interface region reduces the interfacial tension as well as the viscosities of the two fluids, allowing the droplet fluid to penetrate into the carrier fluid forming a droplet.

For efficient heating, the resonator should align with the interface of the two fluids. To demonstrate the feasibility of leveraging microwave heating for droplet generation, the generation process should start with a stable interface. Therefore, the effects of the flow rate ratio between the two phases of the fluids on droplet formation are intentionally eliminated. Integration pressure driven flow and microwave heating for droplet generation justifies an extensive numerical and experimental study which is beyond the scope of this study. When the interface is stable, a pressure balance exists at the interface such that $P_{\mathrm{d}}-P_{\mathrm{c}}=\Delta P$ where $P_{\mathrm{d}}(\mathrm{Pa})$ and $P_{\mathrm{c}}(\mathrm{Pa})$ represent the pressures at the interface from the droplet and carrier fluid side respectively, and $\Delta P=\gamma(2 / H+2 / W)$ is the Laplace pressure where $\gamma(\mathrm{N} / \mathrm{m})$ is the interfacial tension and $H(\mathrm{~m})$ and $W(\mathrm{~m})$ are the channel height and width, respectively. Droplets can be generated by breaking this 
balance such as increasing the applied pressure drop or reducing the Laplace pressure via microwave heating, which are both influenced by the interface position in relevance to the $\mathrm{T}$-junction. Understanding the required applied pressure drop for generating droplets also provides the guidance in microwave heating mediated droplet generation by predicting the power required.

For simplicity, the pressure applied to the inlet of the carrier fluid is kept constant (i.e., $300 \mathrm{mbar}$ ), and the pressure applied to the inlet of the droplet fluid is varied to maintain a stable interface at different positions. It is first estimated based on the pressure balance, $P_{\mathrm{d}}-P_{\mathrm{c}}=\gamma(2 / H+2 / W)$, and the hydrodynamic resistance of each channel ${ }^{31}$ and then slightly adjusted based on the pressure source and tubing during the experiments. In this study, it is varied from 150 to $155 \mathrm{mbar}$ to maintain the interface position from 150 to $1000 \mu \mathrm{m}$ measured from the top of the horizontal channel, as shown in Figure 2.

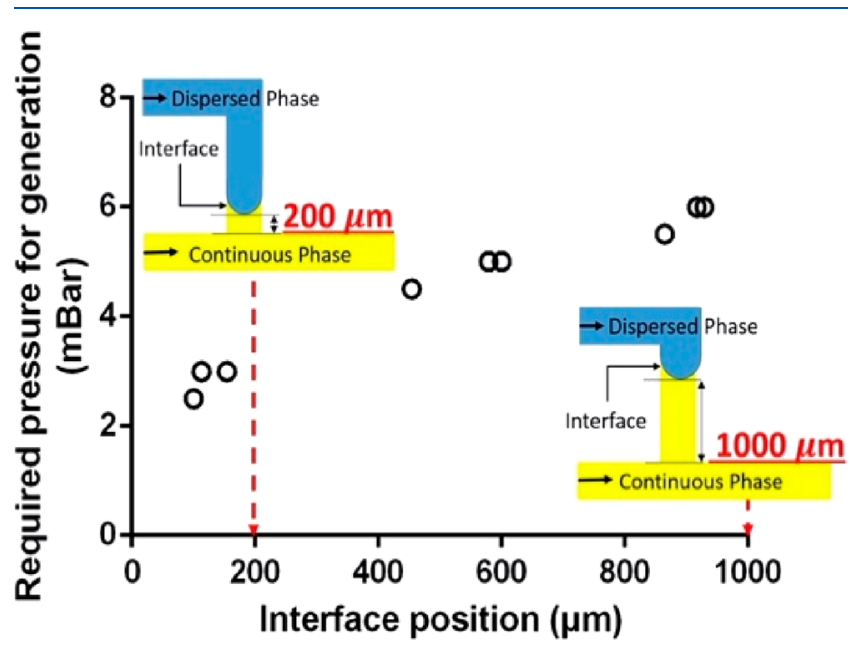

Figure 2. Relation between interface position and pressure difference with the microwave heater

The required pressure increase to generate droplets from a stable interface position of $150 \mu \mathrm{m}$ is around $3 \mathrm{mbar}$, which increases to $6 \mathrm{mbar}$ when the interface is further away from the T-junction, such as $1000 \mu \mathrm{m}$. This information provides guidance in the design and operation of a microwave heater for droplet generation.

In this note, the temperature-dependent interfacial tension was reduced by increasing the temperature of the fluids near the interface using microwave heating while the applied pressures are kept constant. The interfacial tension for the fluid pair (i.e., DI water and FC-40) is $0.052 \mathrm{~N} / \mathrm{m}$ at room temperature. $^{32}$ A Laplace pressure reduction of $4.7 \mathrm{mbar}$ is realized by applying $27 \mathrm{dBm}$ microwave heating as a result of the decreasing interfacial tension. ${ }^{33}$ The calculation is based on Young-Laplace equation $\Delta p=-\gamma\left(\frac{1}{R_{1}}+\frac{1}{R_{1}}\right)$, in which $R_{1}$ and $R_{2}$ are equal to half of the channel width $210 \mu \mathrm{m}$ and height 40 $\mu \mathrm{m}$, respectively, and $\gamma$ is the interfacial tension change in this case to calculate the Laplace pressure difference. When applying $27 \mathrm{dBm}$ microwave heating, the temperature can increase to around $80{ }^{\circ} \mathrm{C}$ corresponding to the interfacial tension change of $7.87 \mathrm{e}^{-3} \mathrm{~N} / \mathrm{m} .{ }^{33}$ The change of Laplace pressure is comparable with the required pressure difference when the resonator is positioned close to the junction, as shown in Figure 2. It suggests that microwave heating has the ability to induce droplet generation by changing the interfacial tension. It should be noted that the fluid viscosity will also decrease with increasing temperature, which would play a role in droplet formation dynamics such as shortening the break-off time. Quantitative evaluation of the role of viscosity on droplet formation dynamics is beyond the scope of this study.

Droplet Generation Process. Microwave heating mediated on-demand droplet generation is demonstrated using a sequence of images shown in Figure 3. Figure 3a shows a

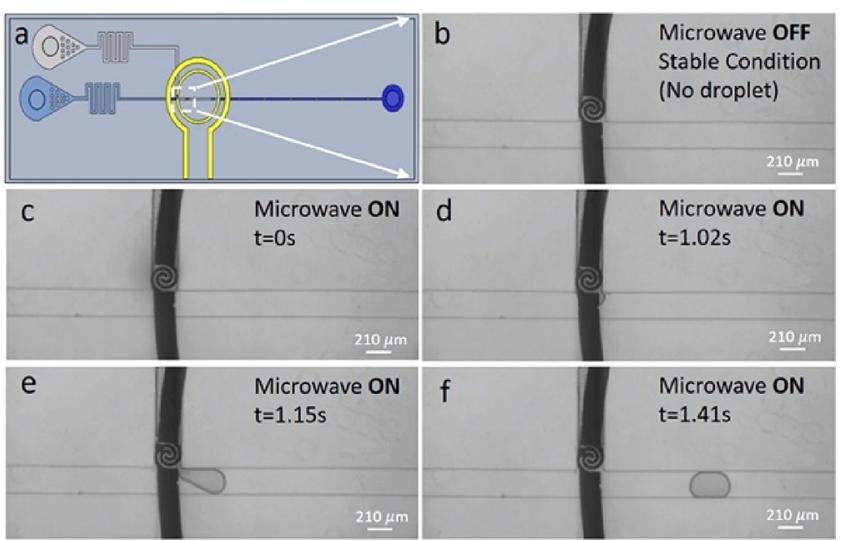

Figure 3. Illustration of time-elapsed droplet generation. (a) Schematic of the chip with the sensor region corresponding to the rest of the images highlighted, (b) microwave heater is off with a stable interface maintained (hard to see due to the sensor), and (c-f) microwave heater is on with droplets being formed.

schematic of the microfluidic chip and the region corresponding to the images. The droplet generation starts with a static interface maintained by the applied pressures (155 and 300 mbar are applied at the inlet of the droplet fluid and carrier fluid, respectively), while the microwave heater is turned off (Figure $3 \mathrm{~b}$ ). After the heater is turned on with a power of 27 $\mathrm{dBm}$ (Figure 3c), it deposits energy into the fluids near the interface, which aligns with the capacitive gap of the resonator (Figure 1). The elevated temperature in the interface reduces the interfacial tension, and thus, the applied pressure difference overcomes the interfacial tension, forcing the droplet fluid to penetrate into the oil stream (Figure $3 \mathrm{~d}$ ) and to be dispersed into a droplet (Figure 3e, f). The droplet formation can be stopped almost instantaneously by turning off the microwave heater because the heat is localized and the fresh roomtemperature liquid fills in the heater region, lowering the temperature quickly.

Microwave mediated droplet generation is basically thermal capillary effects; therefore, microwave power should influence the droplet formation. It is expected that the droplet generation frequency would decrease with the microwave power decrease. To verify this concept, the microwave power was varied from 24 to $27 \mathrm{dBm}$, which would result in a temperature increase of around $40-60{ }^{\circ} \mathrm{C}$ from room temperature. ${ }^{25}$ Figure 4 depicts the effects of microwave power on droplet generation time. It can be clearly observed that a higher microwave power results in a shorter generation time. This fast change in temperature leads to a fast change in Laplace pressure which facilitates a quick droplet generation process. Therefore, adjusting the power of the microwave could be a strategy for controlling droplet generation rate. However, it should be noted that too high a temperature could 
Influence of Microwave power

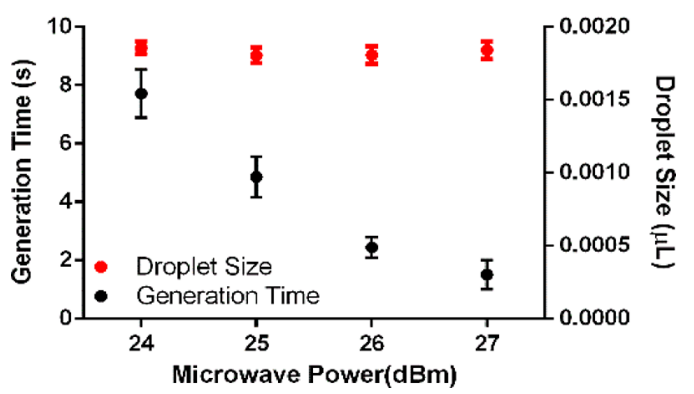

Figure 4. Relation between microwave power and droplet generation time and droplet size

potentially overheat the coated thin PDMS film, causing it to peel off from the heater and influence droplet generation.

The microwave frequency also influences the droplet formation. The power deposition within a droplet is maximized at the resonance frequency associated with the droplet material. Therefore, for efficient heating, the resonance frequency is always chosen for a given material. In fact, we sense the material first to identify its resonance frequency.

The microwave mediated droplet generation occurs in the squeezing regime, ${ }^{34}$ meaning that the interface grows into the oil stream, causing obstruction to the upstream oil and then continues to grow until touching the horizontal channel wall and blocking the upstream oil. The build-up pressure in the upstream oil then squeezes and pinches off the droplet. In the squeezing regime, for a given set of fluids pairs, the droplet volume is mainly dominated by the applied pressures and the geometric conditions, which were maintained constant. It is expected that the droplet volume would remain roughly the same while microwave heating mainly influences droplet formation cycle. The volume of the generated droplets was measured and calculated as presented in Figure 4, showing that the sizes of droplets are close for each case.

The droplet formation cycle is long with the shortest one being around $1 \mathrm{~s}$, marking a generation frequency of around 1 $\mathrm{Hz}$ or lower. This is low compared with most passive droplet generation methods and some high-throughput active droplet generation methods. However, its advantages of on-demand droplet generation and potential to be integrated with microwave sensing make it uniquely useful for many applications that require dynamic changing of the materials and well-controlled time for reaction. For example, the required microwave power could be tuned based on the materials that can be sensed. The demonstrated on-demand droplet generation method can be used in many advanced applications such as X-ray crystallography. Recent studies have demonstrated that using droplets to deliver samples for interaction with X-ray free-electron laser pulses could save sample waste significantly. ${ }^{35,36}$ The microwave heating induced on-demand droplet generation could be advantageous because the formation frequency could be synchronized with the X-ray laser pulses to further reduce sample waste. Some X-ray freeelectron lasers do require higher droplet formation rates than those demonstrated here, such as $10-120 \mathrm{~Hz},{ }^{35}$ which can be improved by optimizing the microwave resonator design and droplet generator design to improve heating efficiency. In addition, the microwave power can be increased with a precaution of the heat tolerance level of the chip material.

\section{CONCLUSIONS AND FUTURE WORK}

In this note, a simple, new method is reported for on-demand droplet generation mediated by microwave heating, which generates disturbance to the interface by reducing the interfacial tension. The microwave heater is capable of confining power into a small region, realizing localized heating, which is advantageous for controlling droplet generation ondemand because the temperature of the fluid can be increased or decreased quickly. The generation cycle can be controlled by the applied microwave power, and the droplet volume is mainly dependent on the applied pressures for a given set of geometric conditions and fluid pair. The limited throughput of generation can be further improved by optimizing the microwave resonator design and the droplet generator design, which justifies an extensive additional study.

\section{AUTHOR INFORMATION}

\section{Corresponding Author}

Carolyn L. Ren - Department of Mechanical and Mechatronics Engineering, University of Waterloo, Waterloo, Ontario N2L 3G1, Canada; (1) orcid.org/0000-0002-92497397; Email: c3ren@uwaterloo.ca

\section{Authors}

Weijia Cui - Department of Mechanical and Mechatronics Engineering, University of Waterloo, Waterloo, Ontario N2L 3G1, Canada

Gurkan Yesiloz - Department of Mechanical and Mechatronics Engineering, University of Waterloo, Waterloo, Ontario N2L 3G1, Canada; Institute of Materials Science and Nanotechnology, Bilkent University, 06800 Ankara, Turkey

Complete contact information is available at:

https://pubs.acs.org/10.1021/acs.analchem.0c04431

\section{Notes}

The authors declare no competing financial interest.

\section{ACKNOWLEDGMENTS}

The authors acknowledge the funding support from the Natural Science and Engineering Research Council (RGPIN2018-04151).

\section{REFERENCES}

(1) Shields, C. W., IV; Reyes, C. D.; López, G. P. Lab Chip 2015, 15 (5), 1230-1249.

(2) Seemann, R.; Brinkmann, M.; Pfohl, T.; Herminghaus, S. Rep. Prog. Phys. 2012, 75 (1), 016601.

(3) Dolomite Bio. https://www.dolomite-bio.com/ (accessed: December 10, 2020).

(4) Markey, A. L.; Mohr, S.; Day, P. J. Methods 2010, 50 (4), 277281.

(5) Yang, C.-H.; Huang, K.-S.; Lin, Y.-S.; Lu, K.; Tzeng, C.-C.; Wang, E.-C.; Lin, C.-H.; Hsu, W.-Y.; Chang, J.-Y. Lab Chip 2009, 9 (7), 961-965.

(6) Teh, S.-Y.; Lin, R.; Hung, L.-H.; Lee, A. P. Lab Chip 2008, 8 (2), 198-220.

(7) Zhu, P.; Wang, L. Lab Chip 2017, 17 (1), 34-75.

(8) Rossow, T.; Heyman, J. A.; Ehrlicher, A. J.; Langhoff, A.; Weitz, D. A.; Haag, R.; Seiffert, S. J. Am. Chem. Soc. 2012, 134 (10), 49834989.

(9) Courtney, M.; Chen, X.; Chan, S.; Mohamed, T.; Rao, P. P.; Ren, C. L. Anal. Chem. 2017, 89 (1), 910-915. 
(10) Shemer, R.; Magenheim, J.; Dor, Y. Curr. Protoc. Mol. Biol. 2019, 127 (1), e90.

(11) Kaminski, T. S.; Scheler, O.; Garstecki, P. Lab Chip 2016, 16

(12), 2168-2187.

(12) Chong, Z. Z.; Tan, S. H.; Gañán-Calvo, A. M.; Tor, S. B.; Loh, N. H.; Nguyen, N.-T. Lab Chip 2016, 16 (1), 35-58.

(13) Churski, K.; Korczyk, P.; Garstecki, P. Lab Chip 2010, 10 (7), $816-818$.

(14) Chen, C.-T.; Lee, G.-B. J. Microelectromech. Syst. 2006, 15 (6), 1492-1498.

(15) Bransky, A.; Korin, N.; Khoury, M.; Levenberg, S. Lab Chip 2009, 9 (4), 516-520.

(16) He, M.; Kuo, J. S.; Chiu, D. T. Appl. Phys. Lett. 2005, 87 (3), 031916.

(17) Link, D. R.; Grasland-Mongrain, E.; Duri, A.; Sarrazin, F.; Cheng, Z.; Cristobal, G.; Marquez, M.; Weitz, D. A. Angew. Chem., Int. Ed. 2006, 45 (16), 2556-2560.

(18) Malloggi, F.; Gu, H.; Banpurkar, A.; Vanapalli, S.; Mugele, F. Eur. Phys. J. E: Soft Matter Biol. Phys. 2008, 26 (1-2), 91-96.

(19) Baroud, C. N.; Gallaire, F.; Dangla, R. Lab Chip 2010, 10 (16), 2032-2045.

(20) Prakash, M.; Gershenfeld, N. Science 2007, 315 (5813), 832835.

(21) Nguyen, N.-T.; Ting, T.-H.; Yap, Y.-F.; Wong, T.-N.; Chai, J. C.-K.; Ong, W.-L.; Zhou, J.; Tan, S.-H.; Yobas, L. Appl. Phys. Lett. 2007, 91 (8), 084102.

(22) Stan, C. A.; Tang, S. K.; Whitesides, G. M. Anal. Chem. 2009, 81 (6), 2399-2402.

(23) Cordero, M. L.; Burnham, D. R.; Baroud, C. N.; McGloin, D. Appl. Phys. Lett. 2008, 93 (3), 034107.

(24) Park, S.-Y.; Wu, T.-H.; Chen, Y.; Teitell, M. A.; Chiou, P.-Y. Lab Chip 2011, 11 (6), 1010-1012.

(25) Yesiloz, G.; Boybay, M. S.; Ren, C. L. Anal. Chem. 2017, 89 (3), 1978-1984.

(26) Koziej, D.; Floryan, C.; Sperling, R. A.; Ehrlicher, A. J.; Issadore, D.; Westervelt, R.; Weitz, D. A. Nanoscale 2013, 5 (12), $5468-5475$

(27) Abduljabar, A. A. Compact microwave microfluidic sensors and applicator; Cardiff University, 2016.

(28) Issadore, D.; Humphry, K. J.; Brown, K. A.; Sandberg, L.; Weitz, D. A.; Westervelt, R. M. Lab Chip 2009, 9 (12), 1701-1706.

(29) Chen, T.; Li, S.; Sun, H. Sensors 2012, 12 (3), 2742-2765.

(30) Boybay, M. S.; Jiao, A.; Glawdel, T.; Ren, C. L. Lab Chip 2013, 13 (19), 3840-3846.

(31) Glawdel, T.; Elbuken, C.; Ren, C. L. Phys. Rev. E 2012, 85 (1), 016322.

(32) Brewer, B. M.; Shi, M.; Edd, J. F.; Webb, D. J.; Li, D. Biomed. Microdevices 2014, 16 (2), 311-323.

(33) Yakhshi-Tafti, E.; Kumar, R.; Cho, H. J. Int. J. Optomechatronics 2011, 5 (4), 393-403.

(34) Christopher, G. F.; Anna, S. L. J. Phys. D: Appl. Phys. 2007, 40 (19), R319.

(35) Fuller, F. D.; Gul, S.; Chatterjee, R.; Burgie, E. S.; Young, I. D.; Lebrette, H.; Srinivas, V.; Brewster, A. S.; Michels-Clark, T.; Clinger, J. A. Nat. Methods 2017, 14 (4), 443-449.

(36) Echelmeier, A.; Villarreal, J. C.; Messerschmidt, M.; Kim, D.; Coe, J. D.; Thifault, D.; Botha, S.; Egatz-Gomez, A.; Gandhi, S.; Brehm, G. Nat. Commun. 2020, 11 (1), 1-10. 